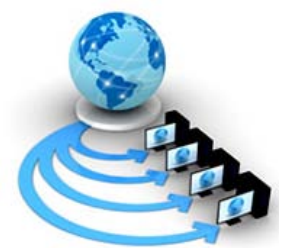

International Journal of Advanced Research in Computer Science

RESEARCH PAPER

\title{
PATTERN RECOGNITION APPROACH FOR FISH MASK SEGMENTATION AND DETECTION FROM VIDEO
}

\author{
Shilpa B Kodli \\ Assistant Professor, \\ Department of MCA, \\ Visvesvaraya Technological University, \\ Post Graduate Centre, Kalaburagi, India
}

\author{
Zeba Juhi \\ PG Scholar, \\ Department of MCA, \\ Visvesvaraya Technological University, \\ Post Graduate Centre, Kalaburagi, India
}

\begin{abstract}
In modern days marine engineering has a dedicated field for fishery monitoring and detection. The most challenging and demanding area for tracking the live fishes under water, where the capturing of images is done in the form of videos but in existing scenario a still image is used to study the features which is a difficult task to overcome this the proposed system is designed and simulated under MATLAB environment for fish region masking and detection to understand and analyze the monitoring study. Here we propose density wise fish area masking and segmentation using video processing for the extraction of appropriate region and analyze the fish.

The major objective of the system is to analyze and understand the importance of region segmentation and extraction under constant monitoring of marine objects. The proposed system targets easy monitoring of fishery services and oceanography study in frame or static image analysis. The system is simulated in such a way that we can obtain the results which reduce the complexity and manual which is a boon for the oceanographic studies.
\end{abstract}

Keywords: novel fish pattern, fish monitoring system, Masking.

\section{INTRODUCTION}

FMS(Fish Monitoring System) also known as vessel monitoring system (VMS) is a general term to depict frameworks that are utilized as a part of business angling to permit ecological and fisheries administrative associations to track and screen the exercises of angling vessels. They are a key portion of checking control and reconnaissance programs at national and worldwide levels. VMS might be utilized to screen vessels in the regional waters of a nation or a subdivision of a nation, or in the Exclusive Economic Zones (EEZ) that develop 200 nautical miles $(370.4 \mathrm{~km}$ ) from the shorelines of numerous nations. VMS frameworks are utilized to enhance the administration and maintainability of the marine environment, through guaranteeing appropriate angling hones and the aversion of illicit angling, and in this way ensure and improve the employments of anglers.

The accurate usefulness of a VMS framework and the related hardware differs with the prerequisites of the country of the vessel's registry, and the territorial or national water in which the vessel is working. Inside local and national VMS activities there are likewise sub-divisions which apply diverse usefulness to various vessel classes.In this context, VMS relates particularly to fisheries administration frameworks. VMS depicts the particular utilization of checking business angling pontoons. It is not to be mistaken for VTS which is portrays the particular utilization of checking marine movement essentially for security and effectiveness in ports and occupied conduits. It is additionally not to be mistaken for particular correspondence advances, for example, AIS, Iridium, Inmarsat, Argos, GPRS which identify with the specialized strategy on which information is transmitted. Diverse VMS frameworks will utilize distinctive correspondence advances relying upon the usefulness prerequisites forced by a national or local VMS activity.The expense of VMS segments will fluctuate as indicated by the usefulness prerequisites of the particular framework being actualized.

All in all the higher the usefulness the more costly the gear and required information join (broadcast appointment costs). The expense of a VMS framework in this manner changes and subsequently the level of government sponsorship (assuming any) shifts as indicated by national and territorial prerequisites. EU and US VMS frameworks require costly installed hardware and a lot of information to be transmitted over satellite connection bringing about high broadcast appointment charges, additionally give an abnormal state of usefulness. In different districts where per vessel cost and immense armada sizes are an issue, correspondence advances, for example, AIS are utilized which essentially decrease hardware and broadcast appointment costs whilst

\section{Project Description}

Each sample in the study is retrieved from an online resource under static frame extraction. Thus the study is concentrated towards the design and analysis of oceanography monitoring and fishery services in understanding the population count.

\section{Problem Statement}

Fish monitoring and fish breeding observations is highly challenging in fishery sciences and ocean eco-system management, and many novel approaches are proposed in retrieving the same but the approaches are appended on a static images captured in the ocean. This creates a delusion 
in evaluation time. Hence a dynamic evaluation of the images is required using underwater video processing.

\section{Objective of Study}

The major objective of the proposed system is to retrieve fish area under segmentation and ROI (Region of Interest) detection for a parallel analysis in a fast and dynamic manner under a framed static image. The overall agenda of proposed system is to create an application with easy portability and reliability for dynamic frame based pattern cum region analysis and extraction.

\section{Scope of the Study}

The proposed system is motivated via the challenges of current scenario under analysis and reflection management for marine object monitoring. The major constrain of repeated density analysis technique is attempted to be resolved under the proposed system design.

\section{Methodology Used}

The system uses a Novel Fish Pattern Detection Algorithm to process the video input which consists of the various steps in processing the images and fetching the output. The parts of algorithm include

$>$ Framing

$>$ Masking

$>$ Pattern Recognition

$>$ Decision Making

Framing is the step where the video is converted into a number of frames depending upon the size of the video. Masking is the part of algorithm where the pixel are analyzed with respect to histogram. Pattern Recognition algorithm focuses on the recognition of pattern using the threshold values. Decision Making takes place once the pattern is recognized for the detected fish present in the frame.

\section{LITERATURE SURVEY}

To provide a stand on the challenge undertaken, a systematic study and its reviews are conducted and archived in this section of paper. Amongst this, the primary focus is towards underwater processing. Underwater PC vision is a subfield of PC vision. Lately, with the advancement of underwater vehicles (ROV, AUV, lightweight planes), that should have the capacity to record and process tremendous measure of data has turned out to be progressively critical.[1]

Applications range from examination of underwater structures for the seaward business to the distinguishing proof and numbering of fishes for natural exploration. Be that as it may, regardless of how enormous the effect of this innovation can be to industry and examination, despite everything it is in an early phase of improvement contrasted with conventional PC vision.[2] One purpose behind this is, the minute the camera goes into the water, a radical new arrangement of difficulties show up. On one hand, cameras must be made waterproof, marine erosion weakens materials rapidly and access and changes to trial setups are exorbitant, both in time and assets. Then again, the physical properties of the water make light carry on in an unexpected way, changing the presence of a same article with varieties of profundity, natural material, streams, temperature and so on.[3]

Using the submerged camera video is also the challenging one. To begin with, the weakening and nonuniformity of Light emitting Diode brightness create numerous closer view items have less diversion from actual foundation and the fish having comparative reaches from the lenses can have necessarily distinctive power.[4]

Then omnipresent disorder is made by non-useful objects, for example, algae the water plants and some wastes dumped into ocean.

Then again, programmed stereo image coordination has been a standout amongst the most intensely examined subjects in computer vision.

All these problems conclude and indicate to use some other technology which is more efficient and more reliable then the still images hence using the underwater submerged cameras to capture the videos using stereo imaging and then processing it using the framework. In this context the captured data from submerged cameras requires more calculations which is solved by the proposed framework[5] is useful to perform the calculations by using the threshold values for the estimation of fish size.

Furthermore, stereo image of the items not only help to setup the video tracker but also helps to find the 3-D space dependable fish length estimation. Then the difficulties of low difference uneven brightening of the cameras can be adjusted and corrected using the Otsu's Thresholding stratergy and also the histogram backprojection system is helpful to track the fishes present in the darker or lighter part of the image.[6]

\section{EXISTING AND PROPOSED SYSTEM}

\section{Existing System}

In the current scenario numerousfish -following frameworks are present for less-complexity and LFR stereo recordings with the operation of a angling trawl-based submerged camera framework. A programmedfish division calculation beats the low-differentiate problem by embracing a histogram backprojection system on twofold neighborhood threshold pictures to guarantee a precise division on thefish shape limits.

\section{Disadvantages of existing system}

The use of a rigid image restricts the use of the images having the moving fishes. This creates a problem to capture still images and increases complexity.

In the existing system only a image can have a single object for processing which is very difficult in real scenario.

The system is more complex and difficult to process. 


\section{Proposed System}

To Build upon a solid component based article coordinating technique, a numerous objective following calculation through a mofled Viterbi information affiliation is anticipated to beat the poor movement congruity and incessant passage/way out fish foc uses under LFR situations. What's more, a computationally feient square coordinating methodology performs effective stereo coordinating that empowers a programmeidh -body tail pay to extraordinarily lessen division mistake and considers an exact fish length estimation.

\section{Module Description}

i Data Acquisition Module:

In this module, we acquire the video files from streaming real time fishes and further storing them in a rational order of standards. Thus in our project we store all the files in .mp4 format.

ii Data Filtering and Organization:

Data acquired is in the form of videos and thus we should reorganize the datasets for processing. Since video processing is complicated we apply framing technique for smaller order frame generation.

iii Segmentation and Object Identification Process

On selecting the frame manually we apply the auto mode detection technique for extracting the objects in the frame. This is the approach for internal collaboration segmentation of the overall images. Basic pattern recognition approach is appended in this step.

iv Masking and Extraction:

Masking is the process of de-attaching the input frame after frame segmentation. Under masking we apply the terminology of detection of ' $n$ ' number of Fishes under various structures and sizes.

\section{System Perspective}

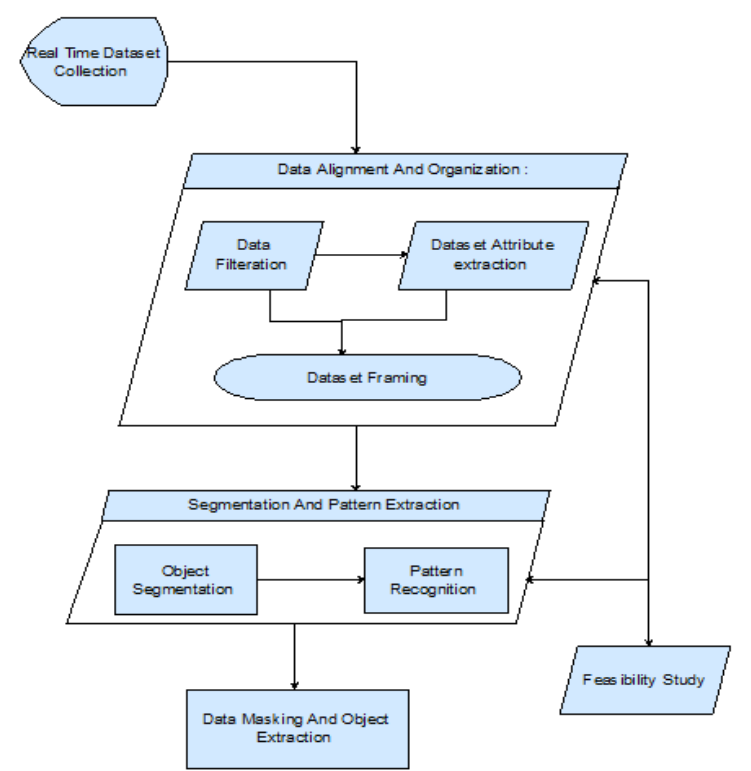

Fig 1. Architecture for the fish mask segmentation and Detection
Above shown figure is the architecture of the fish mask segmentation and Detection. There are three sections in the fish monitoring system. Real time dataset collection, data alignment and organization and segmentation and pattern extraction. In the first section fish images captured in the video will be framed and the static images will be taken as the input to the system. And the collected images will be given as the input to the second section i.e. data alignment and organization. In the second section data filtration and data attribute extraction will be done. Then framing of the obtained images process will take place. Then the processed image will be given as the input to the third section in this section object segmentation and pattern will be recognized. Then the output will be the number of images present in each frame.

\section{RESULTS}

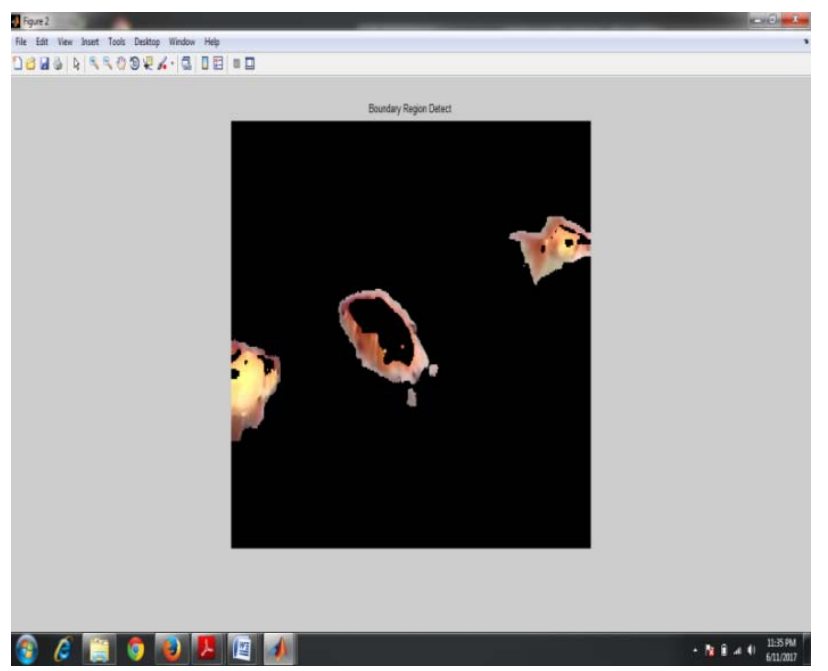

Fig 2. Boundary Region detection and morphing

The figure above illustrates the detection of boundary region and elimination of background by process of morphing. The entire background is removed and the objects are easily available for processing.

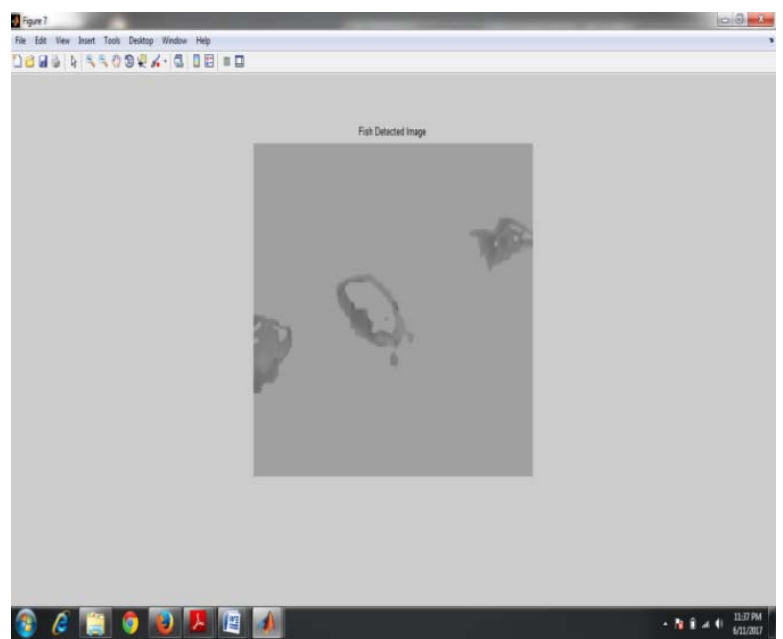

Fig 3. Fish Detected Image

The total number of detected fishes can be seen in the figure above this is the final output of the processing. 


\section{CONCLUSION}

The proposed system is designed and simulated for fish detection under dynamic approach. The video processing makes the proposed system pre-dominant and highlighted for detecting and masking the fishes in live environment. In previous system, the fishes were detected in freezed zone of analysis and hence it was mandatory for ROI to be within the range of analysis, this drawback is overcome in the proposed system by initially retrieving the fishes from video frame, this makes the system more dependable and reliable for processing the fishes.The other drawback which has been overcome is the extraction of fishes was restricted to a domain or a threshold zone of fish's family with internal features. This is now replaced with independent extraction of global features of fishes. In general, the proposed system is more reliable and has higher degree of rigidity compared to the previous system.

\section{FUTURE ENHANCEMENT}

Since some drawbacks have been attempted in the proposed system, a very new level of enhancement can be added in near future such as internal feature recognition and graphically mapping the monitored fishes using advanced robotics and machine learning techniques.

\section{REFERENCES}

[1] K. Williams, R. Towler, and C. Wilson, "Cam-trawl: A combination trawl and stereo camera system," Sea Technol., vol. 51, no. 12, pp. 45-50, 2010.

[2] M.-C. Chuang, J.-N. Hwang, K. Williams, and R. Towler, "Automatic fish segmentation via double local thresh holding for trawl-based underwater camera systems," in Proc. $18^{\text {th }}$ IEEE Int. Conf. Image Process. (ICIP), Sep. 2011, pp. 3145-3148.

[3] M-C. Chu ang, J.-N. Hwang, K. Williams, and R. Towler, "Multiple fish tracking via Viterbi data association for lowframe-rate underwater camera systems ,” in Proc. IEEE Int. Symp. Circuits Syst. (ISCAS), May 2013, pp. 2400-2403.

[4] C. Spampinat o, D. Giordano, R. Di Salvo, Y.-H. J. ChenBurger, R. B. Fisher, and G. Nadarajan, "Automatic fish classification for underwater species behavior understanding," in Proc. 1st ACM Int. Workshop ARTEMIS, 2010, pp. 45-50.

[5] M-C. Chuang, J.-N . Hwang, K. Williams, and R. Towler, "Tracking live fish from low-contrast and low-frame-rate stereo videos,” IEEE Trans. Circuits Syst. Video Technol., vol. 25, no. 1, pp. 167-179, Jan. 2015.

[6] M-C. Chuang, J.-N. Hwang, and K. Williams, "Supervised and unsupervised feature extraction methods for underwater fish species recognition,” in Proc. ICPR Workshop Comput. Vis. Anal. Underwater Imag. (CVAUI), 2014, pp. 33-40. 